\title{
COMPARATIVE STUDY OF QUADRATURE BOOSTER IN DIFFERENT LOCATIONS
}

\author{
Suresh Babu Daram ${ }^{1 *}$, Mallepogu Nagasrinivasulu ${ }^{2}$, Kumar Reddy Cheepati ${ }^{3}$ and \\ P. S. Venkataramu ${ }^{4}$ \\ ${ }^{1}$ Assistant Professor, Dept. of EEE \& Research Coordinator, Centre for Energy, \\ Sree Vidyanikethan Engineering College, Tirupati, India \\ ${ }^{2}$ PG Student, Dept. of EEE, Sree Vidyanikethan Engineering College, Tirupati, \\ India \\ ${ }^{3}$ Research Scholar, EEE, JNTUK \& Assistant Professor, Dept. of EEE, Sree \\ Vidyanikethan Engineering College, Tirupati, India \\ ${ }^{4}$ Dean Academics, Presidency University, Bangalore, India \\ 1* sureshbabudaram@gmail.com, ${ }^{2}$ nagasrinivasulu22@gmail.com
}

\begin{abstract}
Among latest Flexible Alternating Current Transmission System (FACTS) devices, Quadrature Booster $(\mathrm{QB})$ is one of them. QB has the ability to control the voltage magnitude and voltage angle at the buses. The power flow in transmission lines can be controlled in the system. In this paper, the quadrature booster is used to rewrite the Newton- Raphson load flow technique. QB has been incorporated in different locations and compared without QB in the system. A study on variation of power flow in all transmission lines and voltages at all buses for different values of QB control parameters has been carried out on standard 2 machine 5 bus system. MATLAB environment is used for simulation purpose.
\end{abstract}

Keywords - Power Flow Analysis, Modified Jacobian, FACTS, Quadrature Booster

\section{INTRODUCTION}

Electrical power system is one of the man-made complex systems in the world. Based on the electricity generated, it has to be transmitted through the transmission lines. The two power components which are flowing in the line are real power and reactive power. In a three phase ac power system, the power from generating station to the load is transferred through different transmission lines [1]. Power flow is the flow of active power and reactive power in transmission line. The power flow studies give the representation and a systematic mathematical approach for finding the bus voltage magnitude and the angles for the same. The computation of active and reactive power flow can be carried in various transmission lines. The operating condition through steady state situation of a power system is considered for variety of situations and the information is provide to the power engineers to react in an appropriate manner [2].

Flexible AC Transmission systems (FACTS) controllers are used to control the power system steady state difficulties. However, FACTS controllers are used for the enhancement of power system stability. The major role of the FACTS controllers is to control the power flow or load flow in the system. Some of the FACTS devices are static VAR compensator (SVC), Static Synchronous Compensator (STATCOM), Static Series

Received: March 18, 2019

Reviewed: May 21, 2019

Accepted: May 31, 2019

* Corresponding Author 
Synchronous Compensator (SSSC), Thyristor Controlled Series Capacitor (TCSC), Thyristor Switched Series Capacitor (TSSC), Unified Power Flow Controller (UPFC), Interline Power Flow Controller (IPFC), Phase Angle Regulator (PAR), Quadrature Booster (QB) [3-5].

The performance of $\mathrm{QB}$ is comparatively equal performance as Phase Angle Regulator. The power angle in the transmission lines can be controlled by PARs, but QBs are utilized in the line for injecting or boosting the voltage in an appropriate transmission line [6]. The QB requires two transformers units, one at the shunt of the input bus and the other in the series with the transmission line bus [7-8]. Tap changer is used with shunt transformer to exact a component of the system voltage, typical in the range values of positive and negative $20 \%$ of the nominal voltage. From the shunt transformer, the voltage is induced $90^{\circ}$ to the system base voltage with respect to the series transformer. In main transmission circuit the series transformer is connected in series with the line [9].

The remaining discussion of this paper is as follows: The static modelling with basic concept of QB is discussed in section 2. Section 3 gives the developed Jacobian matrix with QB. Section 4 provides the detailed proposed algorithm with QB. The case study and the discussion on the obtained results are given in section 5. Section 6 concludes the overall work carried out for this paper.

\section{STATIC MODELING OF QUADRATURE BOOSTER}

The static modelling of QB is discussed in this section. The QB representation is shown in Fig.1. The electrical representation of QB with the transformer in series with the transmission line impedance is shown in below Fig.2. QB is connected between two buses i \& j.

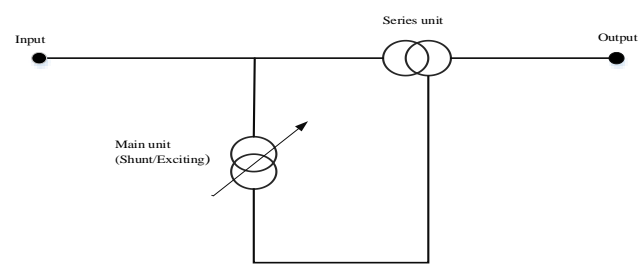

Fig. 1 QB representation

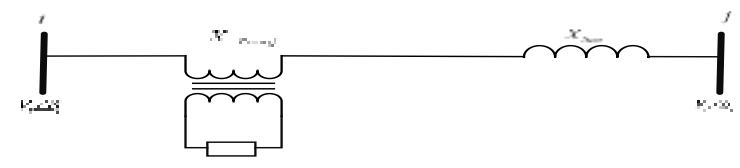

Fig. 2 Representation of Quadrature Booster in the Transmission Line

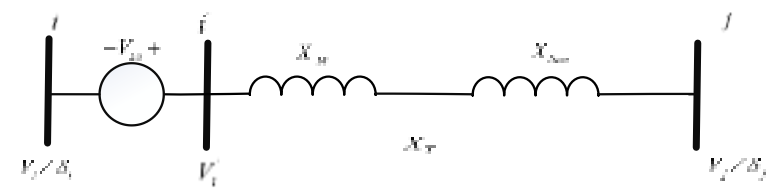

Fig. 3 Representation of Quadrature Booster with Series Voltage Source

Based on the injected voltage from the transformer, the series voltage source is connected with the transformer reactance as shown in Fig.3. The line reactance and the transformer reactance are given separately in the Fig.3. Fig.4 describes that the voltage source in series with the total impedance i.e., the summation of line reactance and the transformer reactance. The equivalent circuit of QB in terms of current source is given in Fig. 5. 


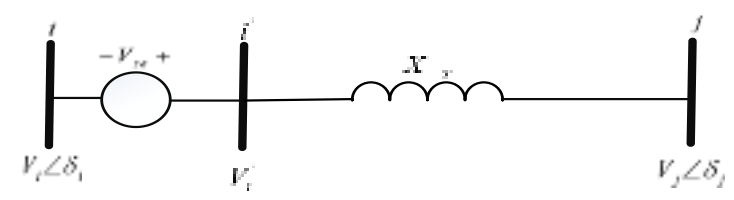

Fig. 4 Representation of Quadrature Booster Voltage Source in Series with the Total Impedance

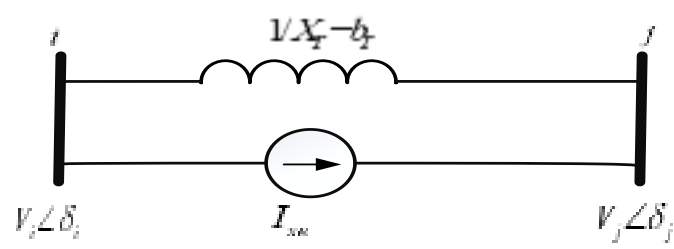

Fig. 5 Equivalent Representation of Quadrature Booster in Current Source

The series voltage of QB in terms of both magnitude and phase angle is given as in Equation (1)

$V_{s e}=r V_{i} e^{j r}$

Where the parameters $r \& \gamma$ is given by $0 \leq r \leq 0.1$ and $-\pi \leq \gamma \leq \pi$.

An imaginary voltage behind the series reactance $X_{s e}$ is given by Equation (2). The phasor diagram of the voltage enhancement can be visualized in Fig. 6.

$V_{i}^{\prime}=V_{s e}+V_{i}$

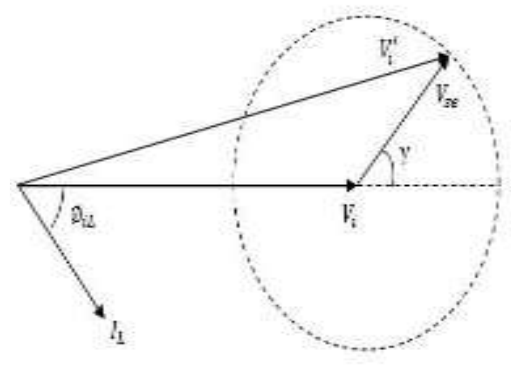

Fig. 6 Phasor Diagram

Voltage at bus-i bus is considered as reference voltage i.e. $V_{i}=V_{i} \angle \mathrm{O}$.

Current in the line is given in Equation (3).

$I_{s e}=-j b_{s e} V_{s e}$

The active and reactive power from the $\mathrm{i}^{\text {th }}$ bus

$P_{i(i n j)}=-V_{i}^{2} b_{s e} r \sin \gamma$

$Q_{i(i n j)}=-V_{i}^{2} b_{s e} r \cos \gamma$

The active and reactive power from the $\mathrm{j}^{\text {th }}$ bus

$P_{i(i n j)}=-V_{i}^{2} b_{s e} r \sin \gamma$

$Q_{i(i n j)}=-V_{i}^{2} b_{s e} r \cos \gamma$ 


\section{MODIFIED JACOBIAN EQUATION OF NEWTON-RAPHSON FLOW SOLUTION}

By neglecting the higher order terms and expanding them, the set of linear expressions is shown in matrix form as shown in equation (8).

$\left[\begin{array}{c}\Delta P \\ \Delta Q\end{array}\right]=\left[\begin{array}{cc}H^{0} & L^{0} \\ M^{0} & N^{0}\end{array}\right]\left[\begin{array}{l}\Delta \delta \\ \Delta V\end{array}\right]$

\subsection{TEXT FONT OF SUB-MATRIX H,L,N}

The diagonal elements for submatrix $\mathrm{H}$ are given below:

$H_{i i}^{1}=H_{i i}^{0} ; \quad H_{j j}^{1}=H_{j j}^{0}-Q_{j(i n j)}$

The off-diagonal elements for submatrix $\mathrm{H}$ are given below:

$H_{i j}^{1}=H_{i j}^{0} ; \quad H_{j i}^{1}=H_{j i}^{0}+Q_{j(i n j)}$

The diagonal elements for submatrix $\mathrm{L}$ are given below:

$L_{i i}^{1}=L_{i i}^{0}+2 P_{i(i m j)} ; \quad L_{j j}^{1}=L_{j j}^{0}+P_{j(i n j)}$

The off-diagonal elements for submatrix L are given below:

$L_{i j}^{1}=L_{i j}^{0} ; \quad L_{j i}^{1}=L_{j i}^{0}+P_{j(i n j)}$

The diagonal elements for submatrix $\mathrm{M}$ are given below:

$M_{i i}^{1}=M_{i i}^{0} ; \quad M_{j i}^{1}=M_{j i}^{0}-P_{j(i n j)}$

The diagonal elements for submatrix $\mathrm{N}$ are given below:

$N_{i i}^{1}=N_{i i}^{0}+2 Q_{i(i n j)} ; \quad N_{j j}^{1}=N_{j j}^{0}+Q_{j(i n j)}$

The off-diagonal elements for submatrix $\mathrm{N}$ are given below:

$N_{i j}^{1}=N_{i j}^{0} ; \quad N_{j i}^{1}=N_{j i}^{0}+Q_{j(i n j)}$

The above equations form the modified Jacobian matrix as shown below.

$$
\left[\begin{array}{c}
\Delta P \\
\Delta Q
\end{array}\right]=\left[\begin{array}{cc}
H^{1} & L^{1} \\
M^{1} & N^{1}
\end{array}\right]\left[\begin{array}{l}
\Delta \delta \\
\Delta V
\end{array}\right]
$$

\section{PROPOSED ALGORITHM}

The proposed algorithm for the computation through the developed mathematical modelling of QB is presented here.

Step-1: $\quad$ Read the data for load flow technique.

Step-2: $\quad$ Compute the admittance matrix from the given line data.

Step-3: $\quad$ Set iteration count as $\mathrm{k} k=0$.

Step-4: Calculate the real and reactive powers with QB in the system.

Step-5: The Jacobian matrix in equation (16) is used for the computation of the real and reactive power mismatch. 
Step-6: $\quad$ Check the power mismatch for the tolerance limit set at the beginning and the results are obtained in the output section. Otherwise go to step 7. Step-7: Calculate the magnitude of voltage and angles variation by using the N-R
method.

Step-8: $\quad$ Modify the load flow equations with QB parameters $r$ and $\gamma$.

Step-9: $\quad$ Set kk=kk+1, next to step 4.

\section{CASE STUDY \& RESULT}

In this section, the effect of quadrature booster on bus voltages \& Power flows has been studied. The model has been tested on various systems and the result of a standard 5-bus system [10-12] is discussed here. In the standard 5-bus system, there are 2 generator buses and 3 load buses and 7 transmission lines. Bus 1 is considered to be slack bus and bus- 2 as generator bus and 3, 4, 5 is load buses. The system's base MVA is 100 MVA and the tolerance limit for converging the power flow solution is considered to be $2 \mathrm{e}^{-10}$. The analysis has been carried out by varying the parameter one at a time keeping the remaining parameter fixed [10]. The suitable value of each parameter for the individual bus system is mentioned below separately. $\gamma$ is varied from 0 to $2 \pi$ and $r$ is varied from 0 to 0.1 .

The Quadrature Booster $(\mathrm{QB})$ is incorporated in the system by considering

1. two load buses (bus-3 \& bus-4).

2. one load bus and one generator bus (bus-2 \& bus-5).

3. both the above combinations (bus- 3 \& bus-4) \& (bus- 2 \& bus-5).

The comparison of placing QB in different locations mentioned above is discussed here. By varying the parameters of $r$ and $\gamma$, we observed several variations in the load bus voltage magnitudes, voltage angles, active $\&$ reactive power flows in the transmission lines and active \& reactive power losses in the system. The suitable values of the parameters for the enhanced voltages, powers and reduced losses are $\gamma=0^{0}, r=0.02$.

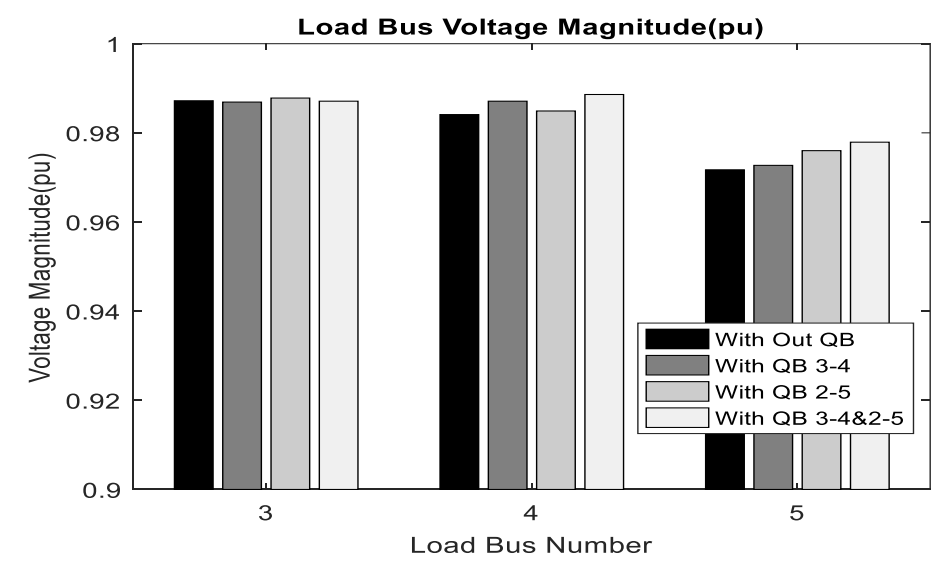

Fig. 7 Load Bus Voltage Magnitude

Fig.7 and Fig.8 shows the voltage magnitudes and voltage angles for the load buses with and without QB respectively. The improvement of the voltage magnitudes and voltage angles at load buses 3,4 \& 5 can be observed by placing QB compared to without QB in the system. After placing QB between buses $3 \& 4$, the voltage magnitude is 
increased at buses 3,4 \& 5 compared to without QB in the system whereas QB between buses $2 \& 5$, the voltage magnitude is increased at buses $3 \& 5$ and decreased at bus 4 compared to QB between buses $3 \& 4$. When QB is placed in both cases like locating between buses $3 \& 4$ and buses $2 \& 5$, the voltage magnitude is increased at buses $3,4 \&$ 5 compared to above two locations in the system.

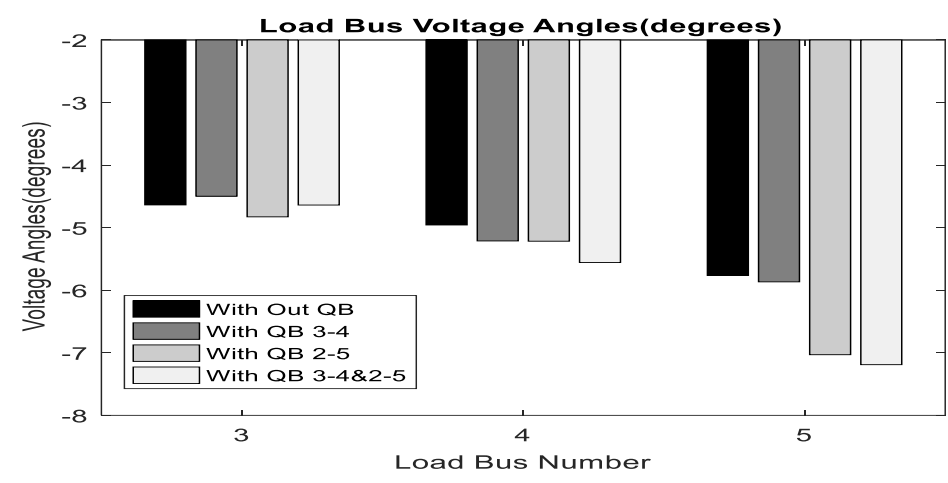

Fig. 8 Load Bus Voltage Angles

After placing QB buses $3 \& 4$, the voltage angle increased at buses $4 \& 5$ and decreased bus 3 compared to without QB in the system whereas QB between buses $2 \&$ 5 , the voltage angle increased at buses $3,4 \& 5$ compared to with QB $3 \& 4$. When QB is placed in both cases like locating between buses $3 \& 4$ and buses $2 \& 5$, the voltage angle increased at buses $3,4 \& 5$ compared to above two locations in the system.

Fig.9 \& Fig.10 shows the active power and reactive powers for the load lines with and without $\mathrm{QB}$ respectively. The improvement of the voltage magnitudes and voltage angles at line 1 to 7 can be observed by placing QB compared to without QB in the system. After placing QB buses $3 \& 4$, the active power increased at lines $1,4 \& 5$ and decreased $2,3,6 \& 7$ lines compared to without QB in the system whereas QB between buses $2 \& 5$, the active power increased at lines 2,3,4,6 \& 7 and decreased line 1 compared to with QB $3 \& 4$. When QB is placed in both cases like locating between buses $3 \& 4$ and buses $2 \&$ 5 , the active power increased at lines $1,2,3 \& 4$ and decreased at line 5,6 \& 7 compared to above two locations in the system.

After placing QB buses $3 \& 4$, the reactive powers increased at lines $1,2,3 \& 7$ and decreased at lines 4,5 \& 6 compared to without QB in the system whereas QB between buses $2 \& 5$, the reactive powers increased at lines $1,4 \& 6$ and decreased at line 2,3,5 \& 7 compared to with QB $3 \& 4$. . When QB is placed in both cases like locating between buses $3 \& 4$ and buses $2 \& 5$, the reactive powers increased at lines $2,4 \& 7$ and decreased at line $3,5 \& 7$ compared to above two locations in the system.

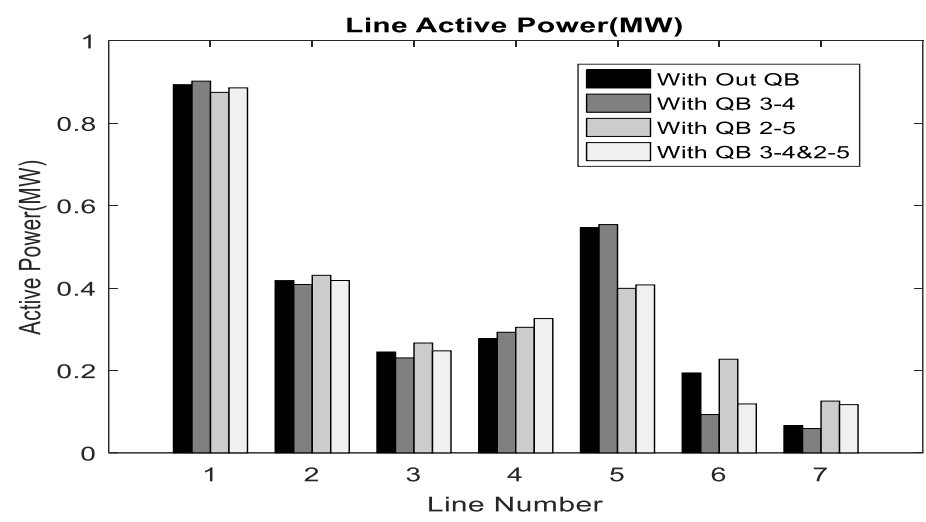

Fig. 9 Active Power Flow in Transmission Line 


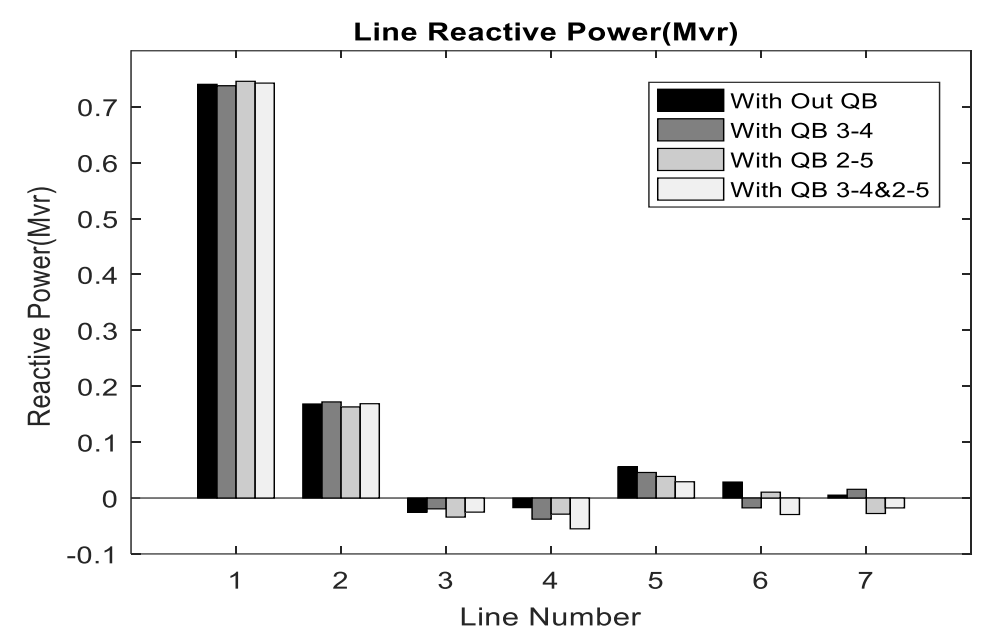

Fig. 10 Reactive Power Flow in Transmission Line

Fig.11 shows the active power loss and reactive power loss for the load bus with and without QB respectively. The more decrement of the active power loss and reactive power loss can be observed by placing QB in the system as comperes with base case. After placing QB buses $3 \& 4$, the active power loss decreased compared to without QB in the system whereas QB between buses $2 \& 5$, the active power loss decreased compared to with QB $3 \& 4$. When QB is placed in both cases like locating between buses $3 \& 4$ and buses $2 \& 5$, the active power loss decreased compared to above two locations in the system.

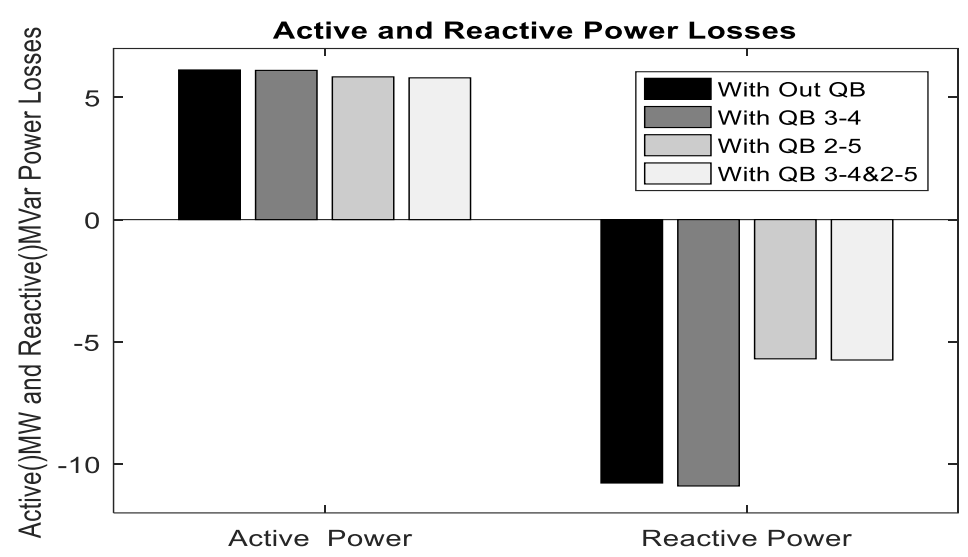

Fig. 11 Active and Reactive power Loss Flow in Transmission Line

\section{CONCLUSIONS}

The power injection model quadrature booster for the transmission line with the modified Jacobian matrix elements has been presented. After incorporating the proposed mathematical model in Newton-Raphson power flow solution, the effects of quadrature booster has been studied. The results of test system shows the potential of the proposed model of quadrature booster and would yield fairly accurate results with regards to bus voltages and power flows. The two control parameters are same for all the three cases considered. In this type of controlling, individual transmission lines are controlled with the shunt connected device with proper phase shifting of 90 degrees. Normally, the impact of QB is be more after placing between two load buses and with a generator bus. The overall system loss is controlled by choosing appropriate values of quadrature booster parameters. 


\section{APPENDIXES}

Nomenclature:

$S$-Apparent power.

$V$-Voltage at particular bus.

$I$-Current in the transmission line.

$\gamma$-Angle at which the voltage has to be injected.

$b$-Susceptance of the transmission line.

$r$-Range of voltage magnitude.

$X$-Reactance of the transmission line.

\section{ACKNOWLEDGMENTS}

The authors are thankful to the management and Centre for Energy, Sree Vidyanikethan Engineering College, Tirupati for the constant encouragement and providing the research facilities to complete this work.

\section{REFERENCES}

[1] Alberto D. Del Rosso and Claudio A. Cañizares. "A Study of TCSC Controller Design for Power System Stability Improvement.” IEEE Transactions on Power Systems, vol. 18, no. 4, November (2003)

[2] Garisapati Dheeraj Krishna, Suresh Babu Daram, P. S. Venkataramu and M. S. Nagaraj. "Effect of Current Based Unified Power Flow Controller under Single Line Outage Condition." IOSR Journal of Electrical and Electronics Engineering, pp 01-07, (2016)

[3] Suresh Babu Daram, P. S. Venkataramu, M. S. Nagaraj. "An Improved Steady-State Model of an Interline Power Flow Controller for the Multi-Transmission System.” International Journal of Grid and Distributed Computing, Vol. 9, No. 5,pp.13-24 , (2016)

[4] Sandeep Sadanandan and Dr. Ghadir Radman. "Power Flow Control using Quadrature Boosters With a suggested Optimal Power Flow Analysis.” IEEE Southeast Con., (2015)

[5] A Al-Riyami,K Burt,P Dyer,P Pretlove,G Manhangwe and S Georgiopoulos. "The Protection of a Quadrature-Booster Connected on the Distribution Network." 12th IET International Conference on Developments in Power System Protection (DPSP), pp.1-5, (2014)

[6] Zhu, M. "Application and Modelling of Quadrature Boosters for the HV Transmission System." International Conference on Power System Technology, August (1998)

[7] Y.J. Fang and D.C. Macdonald. "Dynamic quadrature booster as an aid to system stability." IEE ProcGener. Transm. Distrib., vol. 145, no. I , January (1998)

[8] Luiz A. C. Lopes, Geza Joos and Boon-Teck Ooi. "PWM Quadrature Booster Phase-shifter For FACTS.” IEEE Transactions on Power Delivery, Vol. 11, No. 4, October (1996)

[9] Mallepogu Nagasrinivasulu, Suresh Babu Daram, U.Kamal Kumar and S.Jayachadra, Sairam Seshapalli, P.S.Venkataramu, "Analysis of Power Transmission System using Quadrature Booster." Proccedings of International Conference on Emerging Trends in Engineering (ICECTE), pp.150, March (2019)

[10] William D .Stevenson,Jr. "Elements of Power System Analysis, 4th ed, McGraw-Hill." International edition ISBN 0-07-066584-2, pp.198-199,(1982)

[11] G.W.Stagg, El-Abiad. "Computer Methods in Power Systems Analysis." International Students Edition, McGraw-Hill, Kogakusha Ltd, (1968).

[12] Hadi Sadat. Power System Analysis.: Tata McGraw-Hill Edition (2001) 